\title{
Efficacy and Safety of Acupuncture at Tianshu (ST25) for Functional Constipation: Evidence from 10 Randomized Controlled Trials
}

\author{
Pengfan Li $\mathbb{D}^{1},{ }^{1}$ Yue Luo $\mathbb{D}^{2}{ }^{2}$ Qi Wang, ${ }^{1}$ Shi Shu, ${ }^{1}$ Kanjun Chen, ${ }^{3}$ Donghai Yu $\mathbb{D}^{4}$, \\ and Chunxiang Fan (iD) \\ ${ }^{1}$ Department of Traditional Chinese Medicine, Punan Hospital, Pudong New District, Shanghai 200125, China \\ ${ }^{2}$ Department of Dermatology, Yueyang Hospital of Integrated Traditional Chinese and Western Medicine, \\ Shanghai University of Traditional Chinese Medicine, Shanghai 200437, China \\ ${ }^{3}$ Department of Traditional Chinese Medicine, \\ Shuguang Hospital Affiliated to Shanghai University of Traditional Chinese Medicine, Shanghai 201203, China \\ ${ }^{4}$ Department of Traditional Chinese Medicine, Shanghai Seventh People's Hospital, \\ Shanghai University of Traditional Chinese Medicine, Shanghai 200137, China
}

Correspondence should be addressed to Donghai Yu; yudonghai71@sina.com and Chunxiang Fan; fanxiang1258@163.com

Received 2 August 2020; Revised 24 September 2020; Accepted 30 September 2020; Published 6 November 2020

Academic Editor: Ahmed Mediani

Copyright (c) 2020 Pengfan Li et al. This is an open access article distributed under the Creative Commons Attribution License, which permits unrestricted use, distribution, and reproduction in any medium, provided the original work is properly cited.

Objective. To evaluate the evidence for the efficacy and safety of acupuncture at Tianshu (ST25) for functional constipation (FC). Methods. We systematically searched seven databases to identify randomized controlled trials of acupuncture at ST25 alone or in combination with conventional therapy in the treatment of FC. Risk ratios (RRs) and mean differences (MDs) were calculated using RevMan 5.3 with 95\% confidence interval (CI). Results. The study included ten trials with 1568 participants. Meta-analysis showed that the Cleveland Constipation Score (CCS) for deep needling was significantly lower than that for lactulose (deep needling with low-frequency dilatational wave: $\mathrm{MD}-0.58,95 \% \mathrm{CI}-0.94$ to -0.22 ; deep needling with sparse wave: $\mathrm{MD}-3.67,95 \%$ CI -6.40 to -0.94 ; deep needling with high-frequency dilatational wave: MD $-3.42,95 \%$ CI -5.03 to -1.81$)$. Furthermore, CCS for shallow needling with high-frequency dilatational wave was lower than that for lactulose (MD $-1.77,95 \% \mathrm{CI}-3.40$ to -0.14$)$. In addition, when deep needling was combined with high-frequency dilatational wave, the weekly frequency of spontaneous defecation (FSD) was significantly higher than that for lactulose (MD 1.57, 95\% CI 0.93 to 2.21). Colonic Transit Time (CTT) scores were significantly higher when deep needling was combined with sparse wave (MD $-14.36,95 \%$ CI -18.31 to -10.41 ) or high-frequency dilatational wave (MD $-11.53,95 \% \mathrm{CI}-19.25$ to -3.81 ). The time of first defecation after treatment (TFD) of the shallow needling therapy was significantly longer than that of the lactulose (MD 13.67, 95\% CI 5.66 to 21.67). The CCS 6 months after treatment (CCS6m) for deep needling was significantly lower than that for lactulose (MD $-4.90,95 \%$ CI -5.97 to -3.84 ). Moreover, the FSD 6 months after treatment (FSD6m) for shallow needling was significantly higher than that for lactulose (MD $0.49,95 \%$ CI 0.02 to 0.97 ). The adverse event (AE) rate for lactulose was significantly higher than that achieved with the needling treatments, and this held true for both deep needling therapy (RR 0.41, 95\% CI 0.23 to 0.72) and shallow needling therapy (RR $0.33,95 \%$ CI 0.15 to 0.77 ). Conclusions. The meta-analysis demonstrates that acupuncture at ST25 appears to be more effective than lactulose in the treatment of functional constipation. This was found to be especially true for deep needling with highfrequency dilatational wave, which had a greater impact on improving CCS, FSD, CTT, and CCS6m. Additionally, acupuncture at ST25 was shown to be safer than conventional treatment, with the rate of AE being significantly lower for both deep needling and shallow needling. The trial is registered with https://www.crd.york.ac.uk/prospero/(CRD42019141017)). 


\section{Introduction}

Functional constipation (FC) is a type of general functional gastrointestinal disease. It is characterized by persistent low-frequency defecation, usually accompanied by a tensioning and unpleasant sensation, but without any organic abnormality of the lower abdomen $[1,2]$. FC is a common public health issue, with prevalence of about $15.5 \%$ in North America [3], 14\% in Asia [4], 19.2\% in Europe [5], and $19.7 \%$ in Oceania [5]. FC is not lethal, but it seriously impacts quality of life and carries a heavy financial burden [6-8]. Common methods of FC management include lifestyle modification and pharmaceuticals. Lifestyle modification, such as physical activities and dietary fiber intake, is recommended to promote bowel movement. However, the efficacy of lifestyle modifications still remains uncertain, and the recommendation strength is weak $[9,10]$. Pharmaceuticals which are prescribed to FC patients conventionally, including laxatives, spasmolytics, and gastrointestinal prokinetic agents, have demonstrated efficacy in patients [10]. However, the side effects of the majority of pharmaceuticals, such as dehydration, esophageal obstruction, electrolyte disturbances, and bowel cramps [10], cannot be neglected. Laxatives such as lactulose are often the first-line of defense for FC. Nevertheless, adverse reactions such as flatulence, abdominal pain, nausea, and vomiting are common [11]. Accordingly, seeking a viable alternative cure with less side effects for FC is a priority of both physicians and patients.

Acupuncture is a popular therapy which is often regarded as a complementary and alternative treatment originating from traditional Chinese medicine. It has been used to cure constipation for thousands of years. The efficacy of acupuncture for FC has recently been reported by many high-quality randomized controlled trials (RCTs) [12-16]. Acupuncture can be roughly divided into deep and shallow needling; deep needling, which enters through the peritoneum, can cause severe pain in patients, while shallow needling is not generally painful. Acupuncture is usually combined with a pulse electrotherapy apparatus to strengthen the stimulation of acupoints. Dilatational waves, which are the alternating waveform of sparse waves and dense waves of pulse electric output, have a stronger stimulating effect than sparse waves. However, despite the number of studies, there is still a lack of systematic evaluation of acupuncture depth and waveform in the literature. Tianshu (ST25) [17], a conventional and representative acupoint for constipation [18], has been gaining the growing attention of scholars. Clinical effects are being acknowledged by an increasing number of scholars $[19,20]$. The principal mechanism of acupuncture therapy for FC has also become a major focus in acupuncture research [21, 22]. Several studies have demonstrated that acupuncture's mode of treatment for FC is through modulating peripheral gastrointestinal hormones [23], improving gastrointestinal motility [24], and maintaining the balance of excitatory and inhibitory neurons in the enteric nervous system [25].

The aim of this quantitative research study was to collect evidence about the safety and efficacy of acupuncture at
ST25 for FC to facilitate the clinical application of this treatment.

\section{Methods}

This study was registered with https://www.crd.york.ac.uk/ prospero/(CRD42019141017). It was performed according to the Cochrane Handbook for Systematic Reviews of Interventions [26] and is in accordance with the Preferred Reporting Items for Systematic Reviews and Meta-Analyses (PRISMA) guidelines (Table SM1 in Supplementary Material).

2.1. Literature Search. The search terms "functional constipation," "Dyschezia," and "Colonic Inertia," combined with "Tianshu" and "ST25," were used by two reviewers (PL and YL) in the following databases to search for relevant randomized controlled trials published up to the date of May 5, 2020: PubMed, Embase, the Cochrane Central Register of Controlled Trials (CENTRAL), the China Network Knowledge Infrastructure (CNKI), the Wanfang Data Knowledge Service Platform (Wanfang), the China Biology Medicine disc (CBM), and the China Science and Technology Journal Database (CQVIP). There were no linguistic or geographic restrictions imposed. The detailed search strategy is listed in Table SM2 in Supplementary Material.

2.2. Study Selection. The following inclusion criteria were used to select studies: (i) randomized controlled trials (RCTs), regardless of the use of blinding; (ii) studies including patients with functional constipation diagnosed according to Roman criteria [27], including strenuous defecation, hard defecation, incomplete defecation, obstruction of defecation, defecation in need of manual assistance, and reduction of defecation times (regardless of age, sex, and ethnicity); (iii) studies where the experimental group received acupuncture therapy alone while the control group received any conventional therapy except acupuncture, or where the experimental group received acupuncture therapy in combination with conventional therapies while the control group received the same conventional therapy; (iv) studies where Tianshu (ST25) is used as the exclusive acupuncture method; and (v) studies containing data that was reported regarding therapeutic efficacy and safety.

The exclusion criteria were as follows: (i) articles without data on efficacy or safety; (ii) articles of theoretical explorations, case reports, reviews, and animal studies; and (iii) articles with the full text being unavailable.

2.3. Data Extraction. Four investigators (PL, YL, QW, and SS) independently chose relevant studies after reading the titles and abstracts. Then, the full texts of the selected studies were further assessed. An additional two researchers (KC and DY) created the self-designed data extraction form, which included general information (i.e., the first author, year, location, study design), participant characteristics (i.e., disease duration, average age, sample size), interventions, 
course of treatments, main outcomes, adverse events, and recurrence rates. Any disagreements were settled by discussion among the researchers.

2.4. Methodological Quality Assessment. Four reviewers (PL, $\mathrm{YL}, \mathrm{QW}$, and $\mathrm{KC}$ ) independently assessed the risk of bias in the included studies by applying the Cochrane risk-of-bias tool and Jadad Scale [28]. The Cochrane risk-of-bias tool evaluated the studies using the following parameters [26]: random sequence generation, allocation concealment, blinding of participants and personnel, blinding of the outcome assessment, incomplete outcome data, selective reporting, and other biases. The results were subsequently classified into low risk, high risk, or unclear risk. Additionally, each study was evaluated by the parameters of the Jadad Scale, which includes the following parameters: randomization, double blinding, withdrawals, and dropouts. The Jadad Scale scores range from zero to five points. Studies with a score less than three were considered low-quality trials, whereas those with a score of greater than or equal to three were considered high-quality trials. The final results were cross-checked by two investigators (SS and CF). Any disagreement was settled by discussion between them.

2.5. Data Analyses. Review Manager (RevMan software, version 5.3, Cochrane Collaboration [29]) was used to identify differences in outcome between the experimental (acupuncture) and control groups. Risk ratios (RRs) were calculated for dichotomous data, with a 95\% confidence interval (CI). For continuous data, mean differences (MDs) or standard mean differences (SMDs) were calculated, with a 95\% CI. The degree of heterogeneity between studies was determined using the $I^{2}$ statistic. A fixed model was applied when there was no significant heterogeneity $\left(I^{2}<50 \%\right)$; otherwise, a random effects model was considered suitable. A $P$ value of $<0.05$ was considered statistically significant.

2.6. Outcomes. The primary outcome measured was the Cleveland Constipation Score (CCS) [30] which can comprehensively reflect the constipation condition of the patient and the weekly frequency of spontaneous defecation (FSD) after treatment. First, CCS was divided into the following eight categories [30]: frequency of bowel movements, painful evacuation, incomplete evacuation, abdominal pain, length of time per attempt, assistance for defecation, unsuccessful attempts for evacuation per 24 hours, and duration of constipation. A scoring range of 0 to 4 (except for "assistance for defecation," which used a range of 0 to 2) was derived. Subsequently, the global score was obtained by adding each individual score, and this cumulative score was used to judge the status of constipation comprehensively. Meanwhile, FSD was calculated as the frequency of functional defecation.

Secondary outcomes included the Colonic Transit Time (CTT), the time of first defecation after treatment (TFD), the CCS 6 months after treatment (CCS6m), the FSD 6 months after treatment (FSD6m), the recurrence rate (RER), and the number of adverse events (AE).

\section{Results}

3.1. Selection and Characteristics of Studies. Of the 517 initially retrieved studies, 300 were excluded after abstract and full text reviews on the basis that they were duplicate articles, theoretical explorations, case reports, animal trials, fundamental trials, conferences, or reviews. We eliminated two non-RCTs, 11 mixed interventions, and six studies with overlaps in data. Ultimately, 10 RCTs [31-40] met the inclusion criteria and were included in our systematic review, three of which were unpublished master's theses $[32,34,36]$ (Figure 1). All included studies were performed in China between 2006 and 2018.

The characteristics of the included trials are listed in Table 1. A total of 1568 patients participated in the studies. Of these, 1108 and 460 patients belonged to the experimental and control groups, respectively. All patients met the diagnosis of functional constipation according to Rome III criteria [27]. Eight of the studies [32-39] included two experimental groups (deep needling and shallow needling therapy) and one control group, in which seven studies [33-39] used electroacupuncture stimulation to attach the needle with low-frequency dilatational wave. One study [32] chose the pattern of high-frequency dilatational wave. The remaining two studies $[31,40]$ included an experimental group (deep needling) and a control group, which employed the sparse wave pattern. Control groups for all studies were treated with lactulose. Only one study [32] reported recurrences and calculated RERs. Nine studies [31, 32, 34-40] reported AEs. All studies reported CCS, four studies $[33,35,37,39]$ reported FSD, five studies $[31,32,34,35,40]$ reported CTT, two studies $[32,34]$ reported TFD, three studies $[31,36,40]$ reported CCS6m, and two studies $[32,37]$ reported FSD6m.

3.2. Risk of Bias. Most of the included trials were assessed to be of generally high methodological quality. The risks of bias in all 10 studies are shown in Figure 2. Each study was described as randomized by the authors, and all of them stated methods of random sequences generation, seven of which $[32,35,37,39]$ were based on central randomization, and the remaining three $[31,36,40]$ of which were based on tables of random numbers that were generated by a computer. Only one trial [31] reported allocation concealment which was assigned through an opaque envelope but did not mention blinding method of the outcome assessment. Another trial [39] stated that blinding was not performed on patients or acupuncturists unequivocally, so we judged that the blinding of participants and personnel was high risk for introducing bias. Four trials [32, 34, 36, 39] had a complete and detailed description of final assessment, so we regarded this as having a low risk of bias. Among the remaining trials, allocation concealment, blinding of participants and personnel, and blinding of outcome assessment were not mentioned. Since the protocols of all 10 included trials were not accessible, incomplete outcome data and selective 


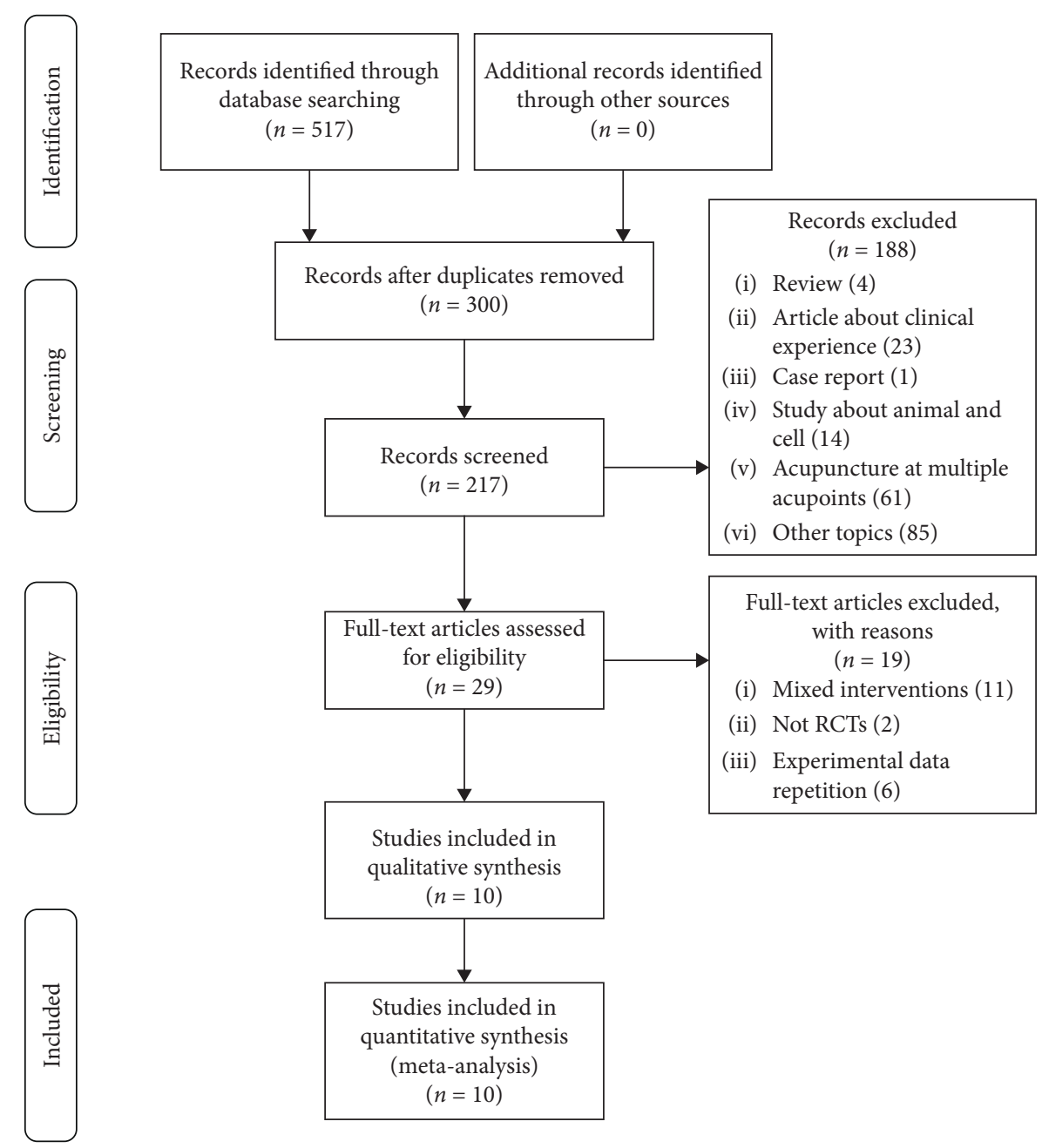

FIGURE 1: Study selection process for a meta-analysis on the safety and efficacy of acupuncture at Tianshu (ST25) for functional constipation.

reporting were generally unclear. Jadad scores of 10 trials ranged from two to five. However, interrater agreement on methodological quality was acceptable (Table 2). The characteristics of participants in the different treatment groups of each study were comparable in terms of baseline (gender, age, severity of disease, etc.).

\subsection{Primary Outcomes}

3.3.1. CCS. Subgroup analysis demonstrated that CCS for deep needling therapy was significantly lower than that for lactulose (deep needling with low-frequency dilatational wave vs. lactulose: $\mathrm{MD}-0.58,95 \% \mathrm{CI}-0.94$ to -0.22 , $P=0.002$; deep needling with sparse wave vs. lactulose: MD $-3.67,95 \%$ CI -6.40 to $-0.94, P=0.008$; deep needling with high-frequency dilatational vs. lactulose: $\mathrm{MD}-3.42,95 \% \mathrm{CI}$ -5.03 to $-1.81, P<0.0001)$. Moreover, CCS for shallow needling with high-frequency dilatational wave was lower than that for lactulose (shallow needling with high-frequency dilatational wave vs. lactulose: $\mathrm{MD}-1.77,95 \% \mathrm{CI}$ -3.40 to $-0.14, P=0.03)$. However, the CCS for shallow needling with low-frequency dilatational wave was comparable with that of lactulose (shallow needling with low- frequency dilatational wave vs. lactulose: $\mathrm{MD} 0.72,95 \% \mathrm{CI}$ -0.39 to $1.83, P=0.21$; see Figure 3 ).

3.3.2. FSD. Subgroup analysis demonstrated that FSD for deep needling with high-frequency dilatational wave was significantly higher than that for lactulose (deep needling with high-frequency dilatational wave vs. lactulose: MD $1.57,95 \%$ CI 0.93 to $2.21, P<0.00001)$. However, the FSD for deep needling with low-frequency dilatational wave and shallow needling therapy was comparable with that for lactulose (deep needling with low-frequency dilatational wave vs. lactulose: $\mathrm{MD} 0.22,95 \% \mathrm{CI}-0.01$ to $0.44, P=0.06$; shallow needling vs. lactulose: $\mathrm{MD}-0.01,95 \% \mathrm{CI}-0.38$ to $0.35, P=0.95$; see Figure 4 ).

\subsection{Secondary Outcomes}

3.4.1. CTT. We used CTT to assess the direct curative effects of the therapies. The trend for CTT was similar to that of FSD. We found that CTT was significantly higher when deep needling was combined with sparse wave or high-frequency dilatational wave (deep needling with 


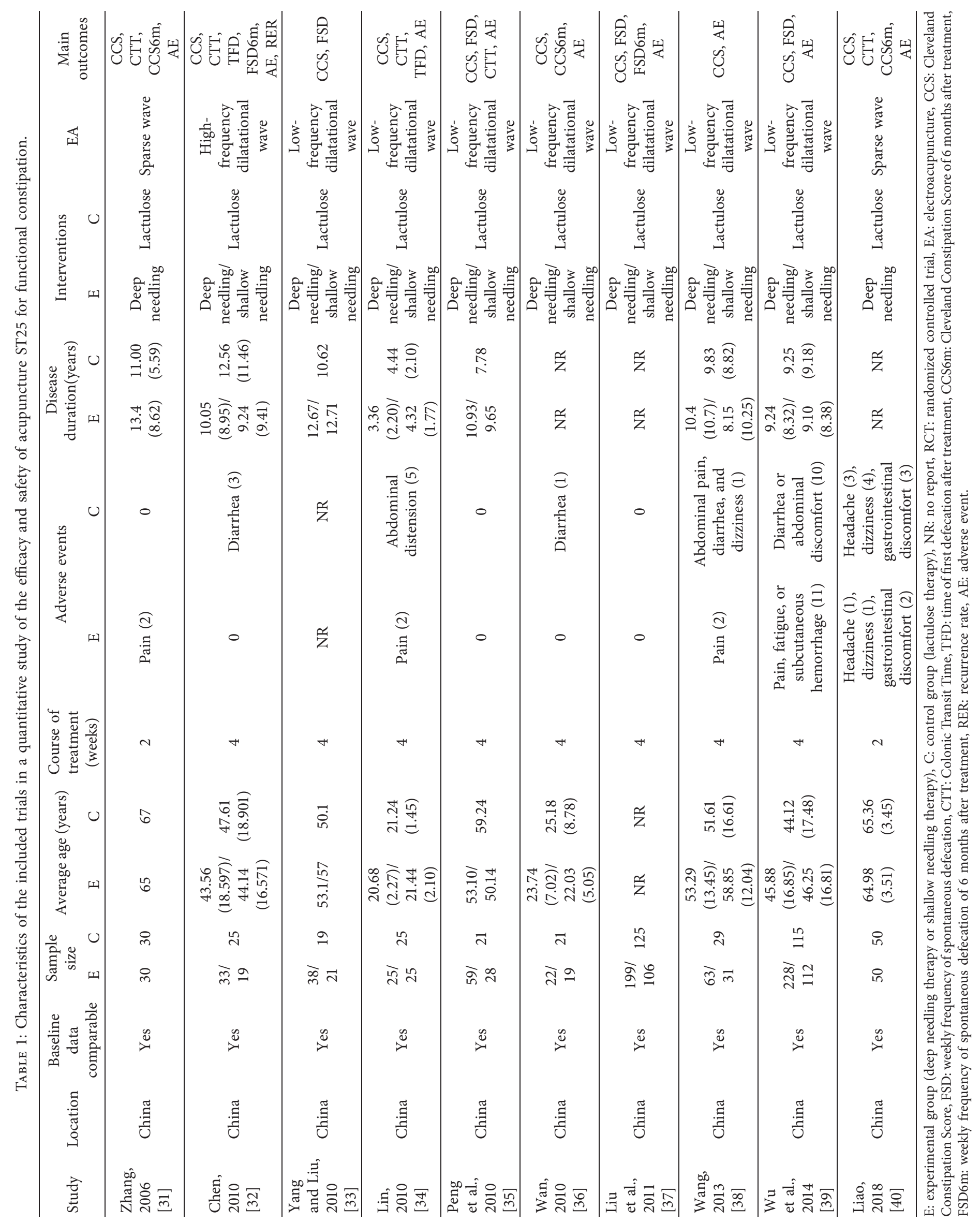



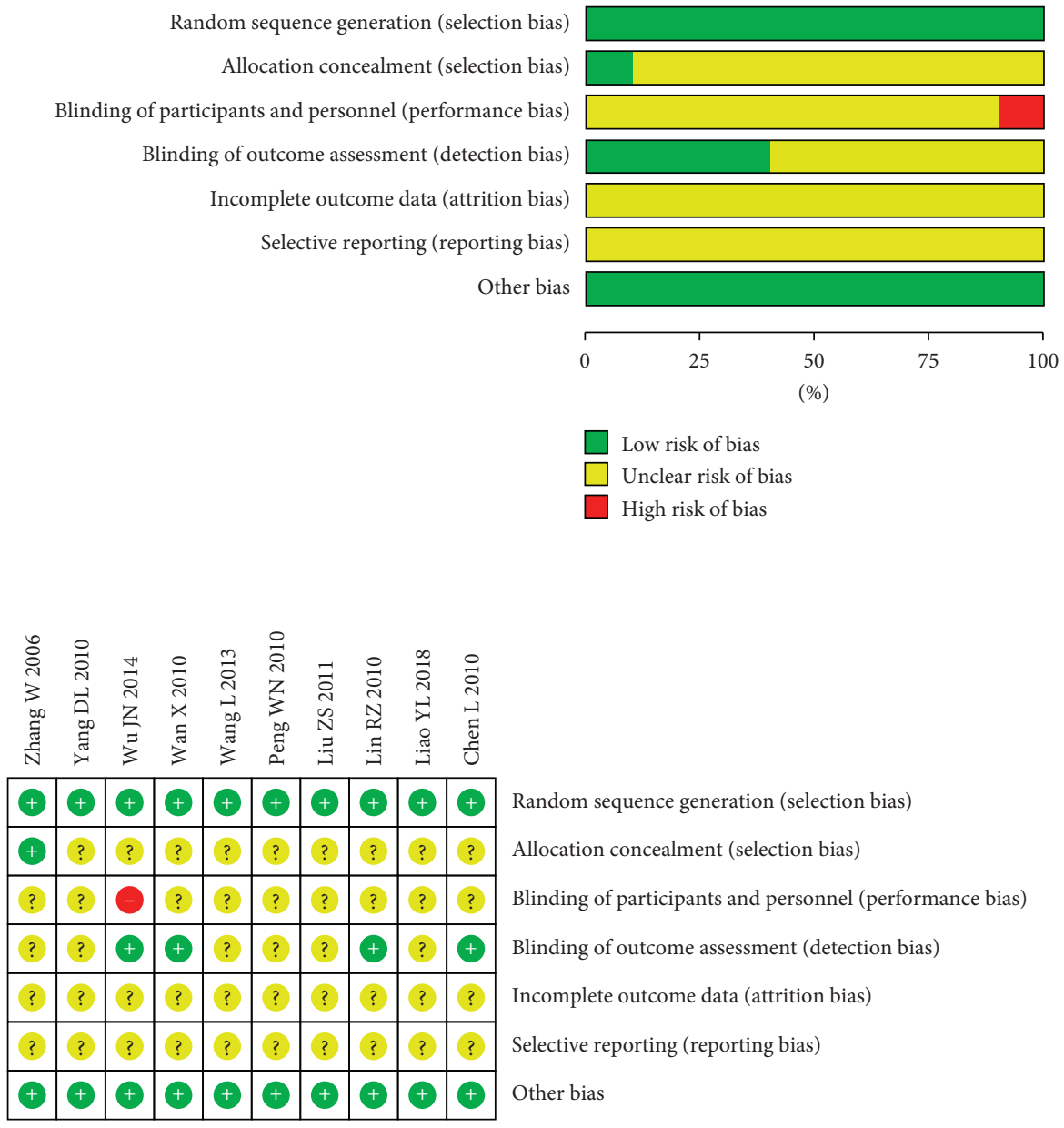

Figure 2: Risk of bias in the studies included in a meta-analysis on the safety and efficacy of acupuncture at Tianshu (ST25) for functional constipation. (a) Risk-of-bias graph. (b) Risk-of-bias summary.

TABLE 2: The Jadad score for assessing risk of bias.

\begin{tabular}{|c|c|c|c|c|}
\hline Study (first author, year) & Random sequence generation & Double blinding & Withdrawals and dropouts & Jadad score \\
\hline Zhang, 2006 [31] & 2 & 0 & 0 & 2 \\
\hline Chen, 2010 [32] & 2 & 1 & 1 & 4 \\
\hline Yang and Liu, 2010 [33] & 2 & 0 & 1 & 3 \\
\hline Lin, 2010 [34] & 2 & 1 & 0 & 3 \\
\hline Peng et al., 2010 [35] & 2 & 0 & 1 & 3 \\
\hline Wan, $2010[36]$ & 2 & 1 & 1 & 4 \\
\hline Liu et al., 2011 [37] & 2 & 0 & 0 & 2 \\
\hline Wang, 2013 [38] & 2 & 0 & 1 & 3 \\
\hline Wu et al., 2014 [39] & 2 & 2 & 1 & 5 \\
\hline Liao, $2018[40]$ & 2 & 0 & 0 & 2 \\
\hline
\end{tabular}

sparse wave vs. lactulose: $\mathrm{MD}-14.36,95 \% \mathrm{CI}-18.31$ to $-10.41, P<0.00001$; deep needling with high-frequency dilatational wave vs. lactulose: $\mathrm{MD}-11.53,95 \% \mathrm{CI}-19.25$ to $-3.81, P=0.003)$. However, the CTTs for deep needling with low-frequency dilatational wave and shallow needling therapy were comparable with that for lactulose (deep needling with low-frequency dilatational wave vs. lactulose: $\mathrm{MD}-2.16,95 \% \mathrm{CI}-8.86$ to $4.54, P=0.53$; shallow needling therapy vs. lactulose: $\mathrm{MD}-2.83,95 \% \mathrm{CI}-8.31$ to 2.65 , $P=0.31$; see Figure 5).

3.4.2. TFD. TFD was used to evaluate the effect time in the different treatment groups. The TFD for deep needling was comparable with that for lactulose (deep needling vs. lactulose: $\mathrm{MD} 3.52,95 \% \mathrm{CI}-0.11$ to $7.16, P=0.06$ ). However, 


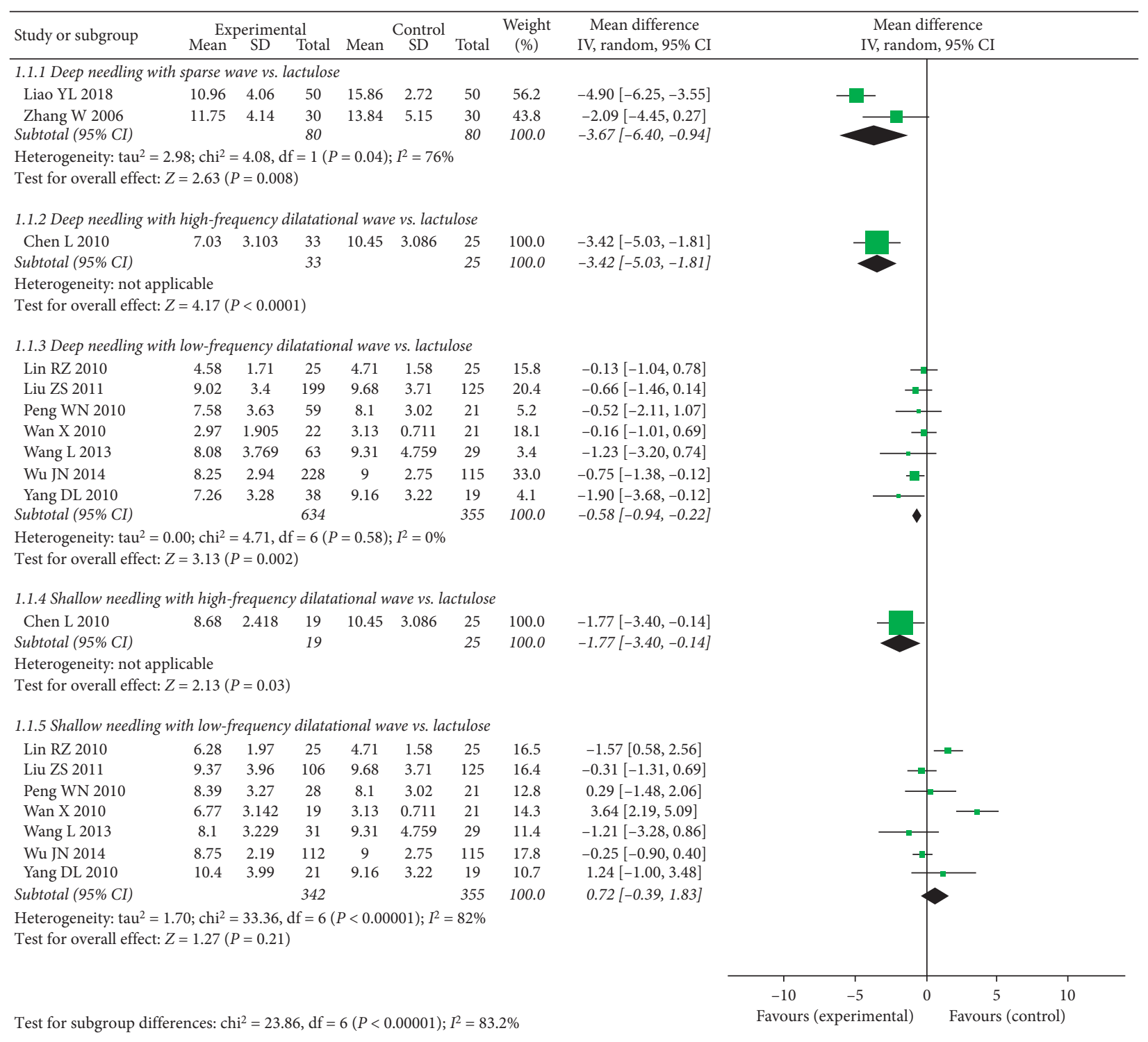

Figure 3: Forest plot comparing Cleveland Constipation Score (CCS) between needling group and control group for patients with functional constipation.

the TFD of the shallow needling therapy was significantly longer than that of the lactulose (shallow needling therapy vs. lactulose: MD 13.67, 95\% CI 5.66 to $21.67, P=0.0008$; see Figure 6).

3.4.3. CCS6m. CCS6m was used to evaluate the long-term effects in the different treatment groups. The CCS6m for deep needling was significantly lower than that for lactulose (deep needling vs. lactulose: $\mathrm{MD}-4.90,95 \% \mathrm{CI}-5.97$ to $-3.84, P<0.00001)$. Nonetheless, the CCS6m of shallow needling was comparable with that of lactulose (shallow needling therapy vs. lactulose: $\mathrm{MD} 0.36,95 \% \mathrm{CI}-2.57$ to 3.29, $P=0.81$; see Figure 7).
3.4.4. FSD6m. Subgroup analysis demonstrated that FSD6m for deep needling was comparable with lactulose treatment (deep needling vs. lactulose: MD 0.64, 95\% CI 0.01 to 1.28 , $P=0.05$ ). However, the FSD6m for shallow needling was significantly higher than that for lactulose (shallow needling vs. lactulose: MD 0.49, 95\% CI 0.02 to $0.97, P=0.04$; see Figure 8).

3.4.5. RER. Only one trial reported RER. Figure 9 shows that there was no significant difference in RER between the experimental and control groups (deep needling vs. lactulose: RR $0.86,95 \%$ CI 0.69 to $1.06, P=0.17$; shallow needling vs. lactulose: RR $0.91,95 \%$ CI 0.72 to $1.15, P=0.43$; see Figure 9). 


\begin{tabular}{|c|c|c|c|c|c|c|c|c|c|c|c|}
\hline Study or subgroup & \multicolumn{3}{|c|}{ Experimental } & \multicolumn{3}{|c|}{ Control } & $\begin{array}{l}\text { Weight } \\
(\%)\end{array}$ & $\begin{array}{l}\text { Mean difference } \\
\text { IV, fixed, } 95 \% \text { CI }\end{array}$ & \multicolumn{2}{|c|}{$\begin{array}{l}\text { Mean difference } \\
\text { IV, fixed, } 95 \% \text { CI }\end{array}$} & \\
\hline \multicolumn{12}{|c|}{ 1.2.1 Deep needling with high-frequency dilatational wave vs. lactulose } \\
\hline Chen L 2010 & 4.89 & 1.326 & 33 & 3.32 & 1.137 & 25 & 100.0 & $1.57[0.93,2.21]$ & & & \\
\hline Subtotal $(95 \%$ CI) & & & 33 & & & 25 & 100.0 & $1.57[0.93,2.21]$ & & & \\
\hline \multicolumn{12}{|c|}{ Heterogeneity: not applicable } \\
\hline \multicolumn{12}{|c|}{ Test for overall effect: $Z=4.85(P<0.00001)$} \\
\hline \multicolumn{12}{|c|}{ 1.2.2 Deep needling with low-frequency dilatational wave vs. lactulose } \\
\hline Liu ZS 2011 & 4.14 & 1.81 & 199 & 3.84 & 1.88 & 125 & 29.5 & $0.30[-0.11,0.71]$ & & - & \\
\hline Peng WN 2010 & 4.08 & 1.37 & 59 & 4.09 & 1.55 & 21 & 9.0 & $-0.01[-0.76,0.74]$ & & & \\
\hline Wu JN 2014 & 4.25 & 1.5 & 228 & 4 & 1.25 & 115 & 56.3 & $0.25[-0.05,0.55]$ & & - & \\
\hline Yang DL 2014 & 4.03 & 1.53 & 38 & 4.21 & 1.93 & 19 & 5.1 & $-0.18[-1.17,0.81]$ & & & \\
\hline Subtotal (95\% CI) & & & 524 & & & 280 & 100.0 & $0.22[-0.01,0.44]$ & & & \\
\hline \multicolumn{12}{|c|}{$\begin{array}{l}\text { Heterogeneity: chi }{ }^{2}=1.16, \mathrm{df}=3(P=0.76) ; I^{2}=0 \% \\
\text { Test for overall effect: } Z=1.91(P=0.06)\end{array}$} \\
\hline \multicolumn{12}{|c|}{ 1.2.3 Shallow needling vs. lactulose } \\
\hline Chen L 2010 & 4 & 1.746 & 19 & 3.32 & 1.137 & 25 & 16.2 & $0.68[-0.22,1.58]$ & & & \\
\hline Liu ZS 2011 & 3.87 & 1.81 & 106 & 3.84 & 1.88 & 125 & 58.1 & $0.03[-0.45,0.51]$ & & & \\
\hline Peng WN 2010 & 3,64 & 1.57 & 28 & 4.09 & 1.55 & 21 & 17.0 & $-0.45[-1.33,0.43]$ & & - & \\
\hline Yang DL 2010 & 3.48 & 2.05 & 21 & 4.21 & 1.93 & 19 & 8.7 & $-0.73[-1.96,0.50]$ & & - & \\
\hline Subtotal (95\% CI) & & & 174 & & & 190 & 100.0 & $-0.01[-0.38,0.35]$ & & & \\
\hline \multicolumn{12}{|c|}{$\begin{array}{l}\text { Heterogeneity: chi }{ }^{2}=4.54, \mathrm{df}=3(P=0.21) ; I^{2}=34 \% \\
\text { Test for overall effect: } Z=0.07(P=0.95)\end{array}$} \\
\hline Test for subgroup d & nces: ch & $\mathrm{i}^{2}=18$ & $1, \mathrm{df}=$ & $2(P<$ & $.0001) ; I$ & $2=89$ & & & $\begin{array}{cc}-2 & -1 \\
\text { Favours (control) }\end{array}$ & $\begin{array}{rr}0 & 1 \\
0 & \text { Favours }\end{array}$ & 2 \\
\hline
\end{tabular}

FIGURE 4: Forest plot comparing the weekly frequency of spontaneous defecation (FSD) between needling group and control group for patients with functional constipation.

\begin{tabular}{|c|c|c|c|c|c|c|c|c|c|}
\hline Study or subgroup & \multicolumn{3}{|c|}{ Experimental } & \multicolumn{3}{|c|}{ Control } & $\begin{array}{l}\text { Weight } \\
(\%)\end{array}$ & $\begin{array}{l}\text { Mean difference } \\
\text { IV, random, } 95 \% \mathrm{CI}\end{array}$ & $\begin{array}{l}\text { Mean difference } \\
\text { IV, random, } 95 \% \mathrm{CI}\end{array}$ \\
\hline \multicolumn{10}{|c|}{ 2.1.1 Deep needling with sparse wave vs. lactulose } \\
\hline Liao YL 2018 & 33.28 & 13.42 & 50 & 47.86 & 12.03 & 50 & 62.5 & $-14.58[-19.58,-9.58]$ & \\
\hline Zhang W 2006 & 33.29 & 13.41 & 30 & 47.28 & 12.05 & 30 & 37.5 & $-13.99[-20.44,-7.54]$ & \\
\hline Subtotal $(95 \%$ CI) & & & 80 & & & 80 & 100.0 & $-14.36[-18.31,-10.41]$ & \\
\hline \multicolumn{10}{|c|}{$\begin{array}{l}\text { Heterogeneity: } \text { chi }^{2}=0.02, \mathrm{df}=1(P=0.89) ; I^{2}=0 \% \\
\text { Test for overall effect: } Z=7.13(P<0.00001)\end{array}$} \\
\hline \multicolumn{10}{|c|}{ 2.1.2 Deep needling with high-frequency dilatational wave vs. lactulose } \\
\hline Chen L 2018 & 40.89 & 7.246 & 14 & 52.42 & 14.94 & 19 & 100.0 & $-11.53[-19.25,-3.81]$ & \\
\hline Subtotal $(95 \% \mathrm{CI})$ & & & 14 & & & 19 & 100.0 & $-11.53[-19.25,-3.81]$ & \\
\hline \multicolumn{10}{|c|}{ Heterogeneity: not applicable } \\
\hline \multicolumn{10}{|c|}{ Test for overall effect: $Z=2.93(P=0.003)$} \\
\hline \multicolumn{10}{|c|}{ 2.1.3 Deep needling with low-frequency dilatational wave vs. lactulose } \\
\hline Lin RZ 2010 & 26 & 18.24 & 25 & 30 & 21.97 & 25 & 35.8 & $-4.00[-15.19,7.19]$ & \\
\hline Peng WN 2010 & 41.6 & 19.58 & 59 & 42.73 & 15.68 & 21 & 64.2 & $-1.13[-9.49,7.23]$ & \\
\hline Subtotal $(95 \%$ CI) & & & 84 & & & 46 & 100.0 & $-2.16[-8.86,4.54]$ & \\
\hline \multicolumn{10}{|c|}{$\begin{array}{l}\text { Heterogeneity: } \text { chi }^{2}=0.16, \mathrm{df}=1(P=0.69) ; I^{2}=0 \% \\
\text { Test for overall effect: } Z=0.63(P=0.53)\end{array}$} \\
\hline \multicolumn{10}{|c|}{ 2.1.4 Shallow needling vs. lactulose } \\
\hline Chen L 2010 & 45.84 & 17.016 & 19 & 52.42 & 14.94 & 19 & 29.0 & $-6.58[-16.76,3.60]$ & - \\
\hline Lin RZ 2010 & 27.6 & 11.7 & 25 & 30 & 21.97 & 25 & 31.6 & $-2.40[-12.16,7.36]$ & \\
\hline Peng WN 2010 & 42.31 & 15.08 & 28 & 42.73 & 15.68 & 21 & 39.4 & $-0.42[-9.15,8.3]$ & \\
\hline Subtotal (95\% CI) & & & 172 & & & 65 & 100.0 & $-2.83[-8.31,2.65]$ & \\
\hline \multicolumn{10}{|c|}{$\begin{array}{l}\text { Heterogeneity: chi }{ }^{2}=0.82, \mathrm{df}=2(P=0.66) ; I^{2}=0 \% \\
\text { Test for overall effect: } Z=1.01(P=0.31)\end{array}$} \\
\hline Test for subgroup & nces: ch & $\mathrm{i}^{2}=16$ & $33, \mathrm{df}=$ & $3(P=0$ & 0.0010); & $=81.6$ & & & 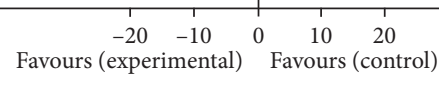 \\
\hline
\end{tabular}

Figure 5: Forest plot comparing Colonic Transit Time (CTT) between needling group and control group for patients with functional constipation. 


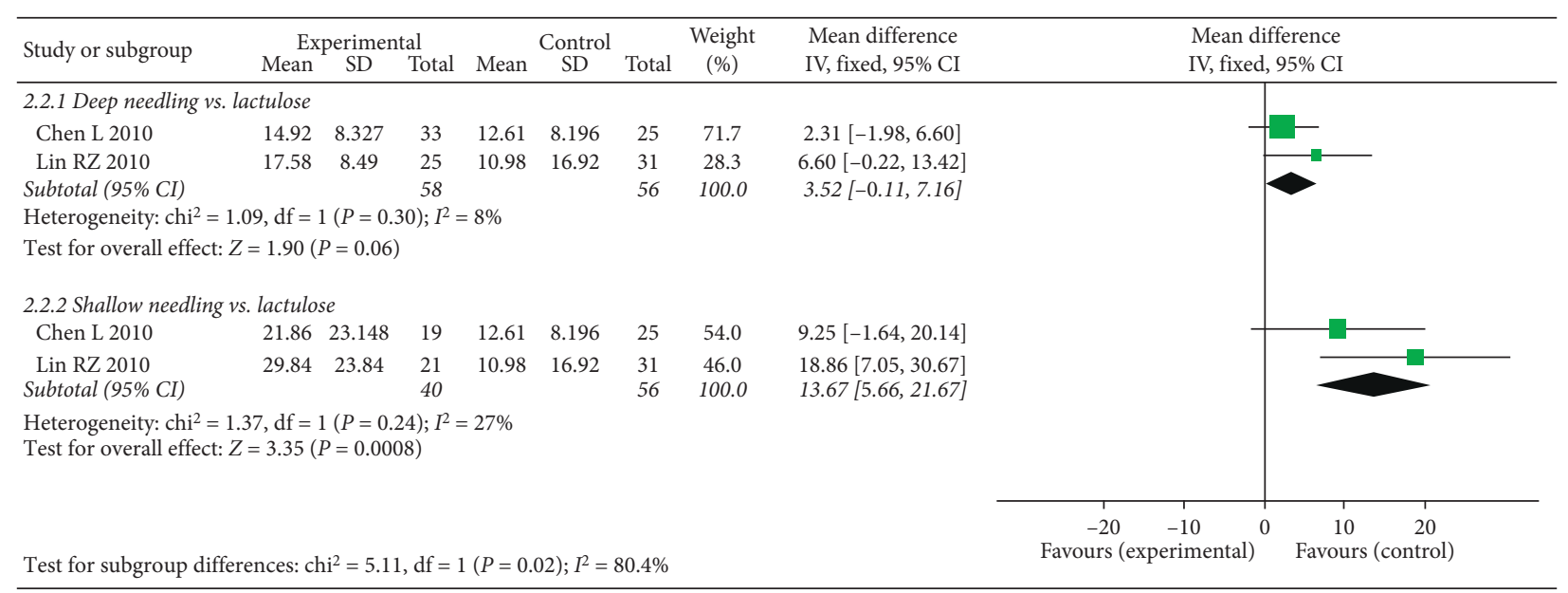

FIGURE 6: Forest plot comparing the time of first defecation after treatment (TFD) between needling group and control group for patients with functional constipation.

\begin{tabular}{|c|c|c|c|c|c|c|c|c|c|c|c|}
\hline Study or subgroup & \multicolumn{3}{|c|}{ Experimental } & Mean & \multicolumn{2}{|l|}{ Control } & $\begin{array}{l}\text { Weight } \\
(\%)\end{array}$ & $\begin{array}{l}\text { Mean difference } \\
\text { IV, fixed, 95\% CI }\end{array}$ & \multicolumn{3}{|c|}{$\begin{array}{l}\text { Mean difference } \\
\text { IV, fixed, } 95 \% \text { CI }\end{array}$} \\
\hline \multicolumn{12}{|c|}{ 3.1.1 Deep needling vs. lactulose } \\
\hline Liao YL 2018 & 10.95 & 4.03 & 50 & 15.86 & 2.72 & 50 & 55.4 & $-4.91[-6.26,-3.56]$ & -1 & & \\
\hline Zhang W 2006 & 10.95 & 4.07 & 30 & 15.84 & 2.7 & 30 & 32.9 & $-4.89[-6.64,-3.14]$ & $\longrightarrow$ & & \\
\hline Subtotal (95\% CI) & & & 80 & & & 80 & 88.3 & $-4.90[-5.97,-3.84]$ & & & \\
\hline \multicolumn{12}{|c|}{$\begin{array}{l}\text { Heterogeneity: } \mathrm{chi}^{2}=0.00, \mathrm{df}=1(P=0.99) ; I^{2}=0 \% \\
\text { Test for overall effect: } Z=9.00(P<0.00001)\end{array}$} \\
\hline \multicolumn{12}{|c|}{ 3.1.2 Shallow needling vs. lactulose } \\
\hline Wan X 2010 & 10.57 & 4.458 & 19 & 10.21 & 5.001 & 21 & 11.7 & $0.36[-2.57,3.29]$ & & & \\
\hline Subtotal (95\% CI) & & & 19 & & & 21 & 11.7 & $0.36[-2.57,3.29]$ & & & \\
\hline \multicolumn{12}{|c|}{$\begin{array}{l}\text { Heterogeneity: not applicable } \\
\text { Test for overall effect: } Z=0.24(P=0.81)\end{array}$} \\
\hline Total (95\% CI) & & & 99 & & & 101 & 100.0 & $-4.29[-5.29,-3.28]$ & & & \\
\hline \multicolumn{9}{|c|}{$\begin{array}{l}\text { Heterogeneity: } \mathrm{chi}^{2}=10.93, \mathrm{df}=2(P=0.004) ; I^{2}=82 \% \\
\text { Test for overall effect: } Z=8.38(P<0.00001) \\
\text { Test for subgroup differences: } \mathrm{chi}^{2}=10.93, \mathrm{df}=1(P=0.0009) ; I^{2}=90.9 \%\end{array}$} & Favours (experimental) & $\begin{array}{l}2 \\
\text { Favour }\end{array}$ & $\begin{array}{l}4 \\
\text { rs (control) }\end{array}$ \\
\hline
\end{tabular}

FIGURE 7: Forest plot comparing the Cleveland Constipation Score of 6 months after treatment (CCS6m) between needling group and control group for patients with functional constipation.

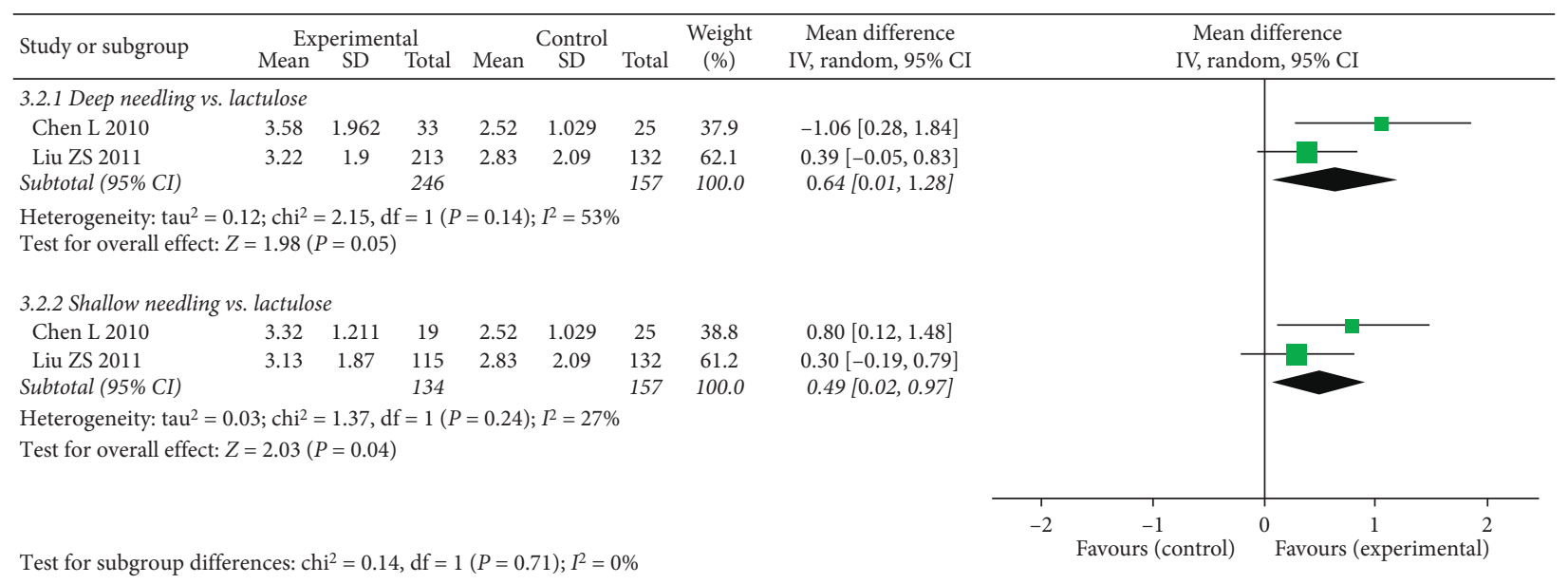

FigURE 8: Forest plot comparing the weekly frequency of spontaneous defecation of 6 months after treatment (FSD6m) between needling group and control group for patients with functional constipation. 


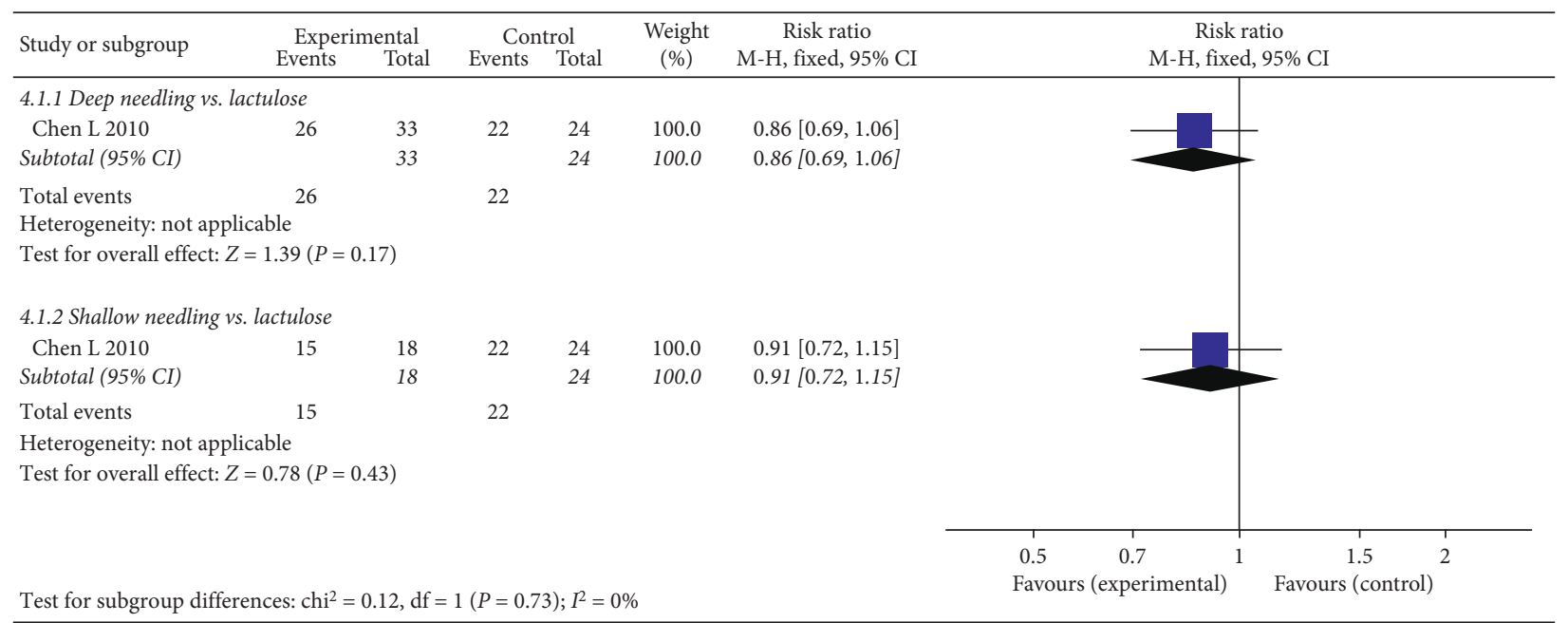

FIGURE 9: Forest plot comparing recurrence rates between needling group and control group for patients with functional constipation.

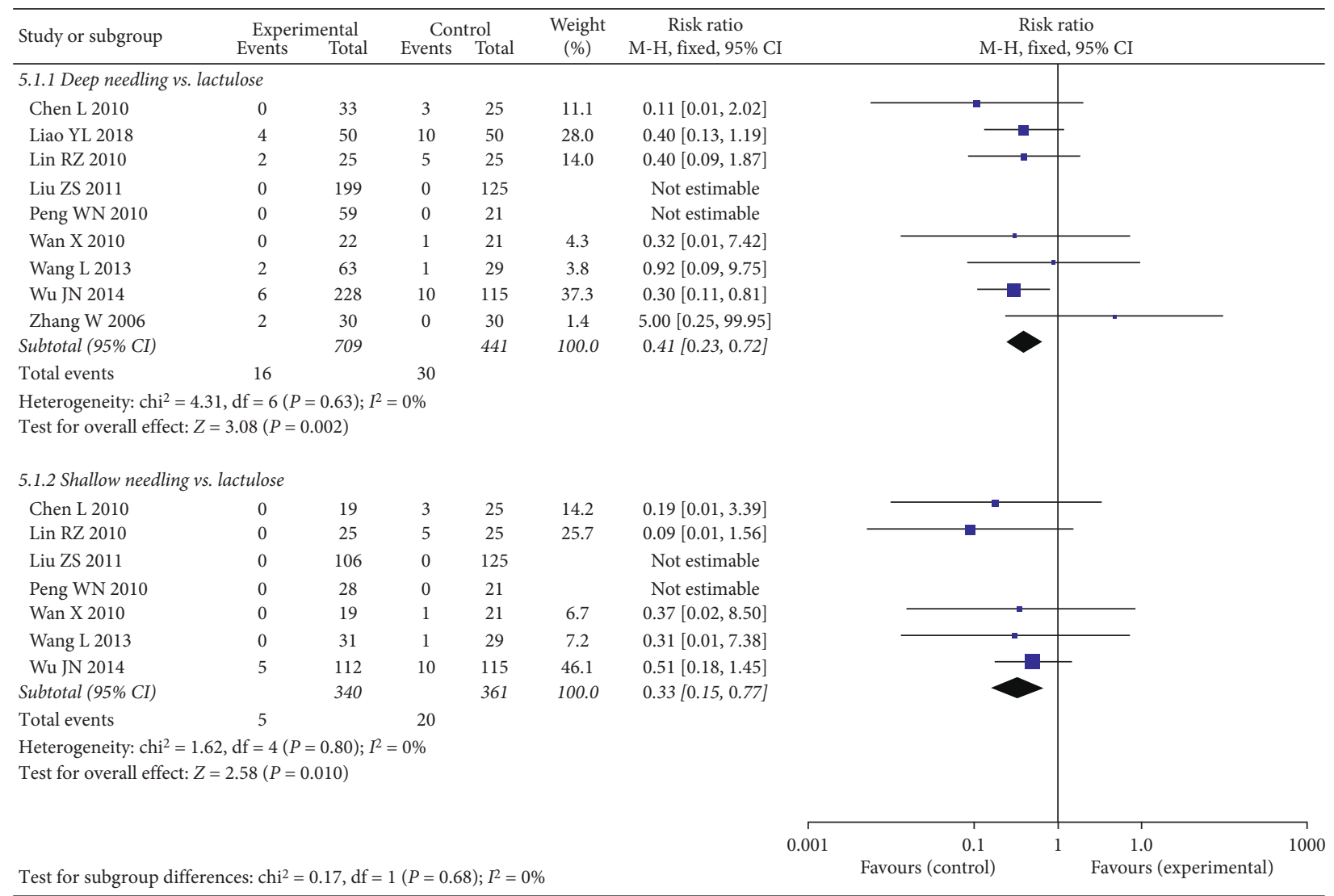

FIGURE 10: Forest plot comparing adverse events between needling group and control group for patients with functional constipation.

3.4.6. AE. The AE rate for lactulose was significantly higher compared to both types of needling treatments (deep needling vs. lactulose: RR $0.41,95 \%$ CI 0.23 to $0.72, P=0.002$; shallow needling vs. lactulose: RR $0.33,95 \%$ CI 0.15 to 0.77 , $P=0.010$; see Figure 10 ).

\section{Discussion}

We were able to demonstrate to a limited extent the safety and efficacy of acupuncture at ST25 for function al constipation, even though the quality of the 10 RCTs which were 
included in the present study was not ideal. Regardless of the waveform of electroacupuncture or the depth of acupuncture, the study was able to comprehensively demonstrate that the CCS for deep needling was significantly lower than that for lactulose (control) group, and thus the condition of the patient was improved. Moreover, the FSD for deep needling with high-frequency dilatational wave was remarkably higher than that for lactulose; however, evidence for this is inadequate because this direct comparison was made in only one study [32]. It is well known that lactulose can increase intestinal osmotic pressure and promote defecation effectively for functional constipation in modern medicine [41]. Nevertheless, the chronic use of lactulose can cause flatus and bacterial adaptation [41-43]. In addition, our study showed that the FSD for lactulose was not significantly different from the frequency for deep needling with low-frequency dilatational wave, shallow needling with high-frequency dilatational wave, or shallow needling with low-frequency dilatational wave.

However, of note is that the FSD significantly increased for deep needling therapy with high-frequency dilatational wave. CTT scores showed a similar trend with a significant improvement, and this was only when deep needling was combined with sparse wave and high-frequency dilatational wave. These findings suggest that deep needling at ST25 may promote defecation by reducing CTT. Modern studies have shown that the nerve segments of the colon are T10 to L3 and the sacral plexuses, and the nerve segment of ST25 is T10 [44]. Additionally, it has been shown that the afferent impulses of acupuncture at ST25 can be projected to the colon [44]. Subsequently, the afferent impulse of acupuncture at ST25 is transmitted through two pathways of the somatic nerve and vascular wall nerve plexus, through the spinal cord, to all the levels of centers of cerebral cortex and connects with the viscera, which then transmit it through the autonomic nervous system and bodily fluid to regulate gastrointestinal function [45]. This significantly increases the amplitude and frequency of the distal colon [46], strengthens the tension of the colon, makes the contraction more powerful, and is thus beneficial to defecation [47].

The long-term effect of defecation improvement is as important as the short-term effect for FC patients. The pathogenesis of FC is mainly related to colonic motility disorder, perianal sphincter dysfunction, and abnormal pathological changes of intestinal hormones. Gastrointestinal hormones mainly include excitatory transmitters such as acetylcholine (ACh) and substance $\mathrm{P}$, as well as inhibitory transmitters such as nitric oxide (NO), vasoactive intestinal peptide (VIP), and adenosine triphosphate (ATP) [36]. Recently, it has been found that interstitial Cajal cell (ICC) is a pacemaker of slow waves in the intestinal tract, which is involved in the process of information transmission in the intestinal noncholinergic nervous system (NANC) and has the potential role of controlling gastrointestinal motility. It is also related to the pathogenesis of human gastrointestinal motor disease $[48,49]$. There was irregular distribution of ICC in the whole colon tissue in patients with functional constipation, and the volume of ICC was significantly reduced [50]. Acupuncture at ST25 can cause severe pain, which triggers a large number of pain receptors, and these excite unmyelinated III (AS) and IV (C) afferent fibers and central nervous system fibers to activate the brainstem. It produces bidirectional regulation of neurophysiology [51] and can regulate increased secretion of neurotransmitters such as acetylcholine ( $\mathrm{ACh}$ ), substance $\mathrm{P}$, and nitric oxide (NO). At the same time, acupuncture stimulation may promote the repair of colonic neuromuscular tissue, especially in the repair of ICC [36]. These comprehensive factors may be the physiological basis of acupuncture at ST25 in the treatment of functional constipation, which is beneficial to patients not only in the short term, but also in the long term.

However, no matter which treatment is used, recurrence cannot be ignored, which is comparable in the control group and the experimental group. This suggests that acupuncture may not increase RER. Although the AE of acupuncture at ST25 was lower than that of lactulose, the AE of pain could not be ignored. The included studies [31, 34, 38-40] showed that the side effects of the needling group could disappear within a short limited period of time after treatment of symptoms or without any special treatment. Future research should explore a combination of multiple acupoints, combined with acupuncture anesthesia technology, to develop a treatment scheme with better curative effect and less pain.

There are some limitations to this study. Firstly, the sample size is not large enough to draw reliable conclusions, and the quality of the included trials was not overwhelmingly high. Of the 10 trials, only one trial [31] reported an allocation concealment method. Additionally, none of the studies involved blinding of the researchers and participants. Secondly, all the studies were conducted in China, and the results may vary according to ethnicity or diet. Thirdly, the number of events in some subgroups that the results were based on was exceedingly small, which may affect the interpretations.

\section{Conclusions}

In conclusion, acupuncture at ST25 may be more effective than lactulose for functional constipation. In particular, deep needling with high-frequency dilatational wave had a substantial impact on improving CCS, FSD, CTT, and CCS6m. In terms of safety, the rate of $\mathrm{AE}$ for acupuncture with both deep and shallow needling was significantly lower than that for lactulose. However, a larger number of high-quality RCTs with a lower risk of bias and adequate sample sizes are required to confirm the results of this quantitative research study.

\section{Abbreviations}

FC: $\quad$ Functional constipation

RR: $\quad$ Risk ratio

MD: $\quad$ Mean difference

SMD: $\quad$ Standard mean difference

CI: $\quad$ Confidence interval

CCS: $\quad$ Cleveland Constipation Score

FSD: Weekly frequency of spontaneous defecation

CTT: $\quad$ Colonic Transit Time 
TFD: $\quad$ The time of first defecation after treatment

CCS6m: Cleveland Constipation Score of 6 months after treatment

FSD6m: Weekly frequency of spontaneous defecation of 6 months after treatment

RER: $\quad$ Recurrence rate

AE: $\quad$ Adverse event

RCT: $\quad$ Randomized controlled trial

CENTRAL: Cochrane Central Register of Controlled Trials

Embase: Excerpta Medica database

CNKI: China Network Knowledge Infrastructure

Wanfang: Wanfang Data Knowledge Service Platform

CBM: China Biology Medicine disc

CQVIP: China Science and Technology Journal

Database

ICC: Interstitial Cajal cell

E: $\quad$ Experimental group

C: $\quad$ Control group

EA: $\quad$ Electroacupuncture

NR: $\quad$ No report.

\section{Data Availability}

The data used to support the findings of this study are included within the article and the supplementary information files.

\section{Disclosure}

The funders played no role in designing, collecting, analyzing, and interpreting data or in writing the article.

\section{Conflicts of Interest}

The authors declare that there are no conflicts of interest regarding the publication of this paper.

\section{Authors' Contributions}

Pengfan Li and Yue Luo contributed equally to this work. CF and DY conceived the study. PL and YL designed the study, performed literature researches, and prepared the original draft. QW, SS, and KC chose relevant studies. PL, YL, QW, and SS extracted data. PL, YL, QW, and KC assessed the quality of trails. PL, YL, QW, SS, and KC analyzed the data. PL, YL, QW, SS, KC, DY, and CF contributed to the revision of the manuscript. All authors read and approved the final manuscript.

\section{Acknowledgments}

This project was supported by grants from Construction of Key Special Diseases of Traditional Chinese Medicine in Pudong New Area of Shanghai (no. PDZY-2018-0618), Clinical Specialty Project of Traditional Chinese Medicine in Pudong New Area of Shanghai (no. PDZY-2018-0612), Academic Leaders Training Program of Pudong Health Bureau of Shanghai (no. PWRD2018-08), and Traditional Chinese Medicine School Pudong Base Construction Project of Shanghai Pudong New Area (Yan's Internal Medicine) (no. PDZY-2018-0704).

\section{Supplementary Materials}

Table SM1: PRISMA checklist. Table SM2: search strategy. (Supplementary Materials)

\section{References}

[1] G. F. Longstreth, W. G. Thompson, W. D. Chey, L. A. Houghton, F. Mearin, and R. C. Spiller, "Functional bowel disorders," Gastroenterology, vol. 130, no. 5, pp. 1480-1491, 2006.

[2] F. Mearin, B. Lacy, and L. Chang, "Rome IV: the functional bowel disorders," Gastroenterology, vol. 150, no. 1, pp. 1393-1407, 2016.

[3] P. D. Higgins and J. F. Johanson, "Epidemiology of constipation in North America: a systematic review," American Journal of Gastroenterology, vol. 99, no. 4, pp. 750-759, 2004.

[4] C. Cheng, A. O. O. Chan, W. M. Hui, and S. K. Lam, "Coping strategies, illness perception, anxiety and depression of patients with idiopathic constipation: a population-based study," Alimentary Pharmacology \& Therapeutics, vol. 18, no. 3, pp. 319-326, 2003.

[5] S. M. Mugie, M. A. Benninga, and C. Di Lorenzo, "Epidemiology of constipation in children and adults: a systematic review," Best Practice \& Research Clinical Gastroenterology, vol. 25, no. 1, pp. 3-18, 2011.

[6] A. Guerin, R. T. Carson, B. Lewis, D. Yin, M. Kaminsky, and $\mathrm{E}$. $\mathrm{Wu}$, "The economic burden of treatment failure amongst patients with irritable bowel syndrome with constipation or chronic constipation: a retrospective analysis of a Medicaid population," Journal of Medical Economics, vol. 17, no. 8, pp. $577-586,2014$.

[7] H. Ansari, Z. Ansari, and T. Lim, "Factors relating to hospitalisation and economic burden of paediatric constipation in the state of Victoria, Australia, 2002-2009," Journal of Paediatric Child Health, vol. 50, no. 12, pp. 993-999, 2014.

[8] B. Wirta, P. Hodgkins, and A. Joseph, "Economic burden associated with chronic constipation in Sweden: a retrospective cohort study," Clinicoecon Outcomes Res, vol. 6, no. 1, pp. 369-379, 2014.

[9] J. E. Shin, H. K. Jung, T. H. Lee et al., "Guidelines for the diagnosis and treatment of chronic functional constipation in Korea, 2015 revised edition," Journal of Neurogastroenterology and Motility, vol. 22, no. 3, pp. 383-411, 2016.

[10] F. Mearin, C. Ciriza, and M. Mínguez, "Clinical practice guideline: irritable bowel syndrome with constipation and functional constipation in the adult," Revista Espanola de Enfermedades Digestivas, vol. 108, no. 6, pp. 332-363, 2016.

[11] T. Piche and M. Dapoigny, "Comparative efficacy and safety of lactulose plus paraffin vs polyethylene glycol in functional constipation: a randomised clinical study," United European Gastroenterology Journal, vol. 8, no. 8, p. 923, 2020.

[12] H. Y. Lee, O. J. Kwon, and J. E. Kim, "Efficacy and safety of acupuncture for functional constipation: a randomised, shamcontrolled pilot trial," BMC Complementary and Alternative Medicine, vol. 18, no. 1, p. 186, 2018.

[13] Z. Liu, S. Yan, J. Wu et al., "Acupuncture for chronic severe functional constipation: a randomized trial," Annals of Internal Medicine, vol. 165, no. 11, pp. 761-769, 2016.

[14] A. G. Klauser, A. Rubach, O. Bertsche, and S. A MüllerLissner, "Body acupuncture: effect on colonic function in 
chronic constipation," Zeitschrift Fur Gastroenterologie, vol. 31, no. 10, pp. 605-608, 1993.

[15] C. Y. Chen, M. D. Ke, C. D. Kuo, C. H. Huang, Y. H. Hsueh, and J. R. Chen, "The influence of electro-acupuncture stimulation to female constipation patients," The American Journal of Chinese Medicine, vol. 41, no. 2, pp. 301-313, 2013.

[16] E. Broide, S. Pintov, S. Portnoy, J. Barg, E. Klinowski, and E. Scapa, "Effectiveness of acupuncture for treatment of childhood constipation," Digestive Diseases and Sciences, vol. 46, no. 6, pp. 1270-1275, 2001.

[17] WHO Regional Office for the Western Pacific, WHO Standard Acupuncture Point Locations in the Western Pacific Region, WHO Regional Office for the Western Pacific, Manila, Philippines, 2008.

[18] J. Yin and J. D. Z. Chen, "Gastrointestinal motility disorders and acupuncture," Autonomic Neuroscience, vol. 157, no. 1-2, pp. 31-37, 2010.

[19] H. Zheng, J. Xu, X. Sun et al., "Electroacupuncture for patients with refractory functional dyspepsia: a randomized controlled trial," Neurogastroenterology \& Motility, vol. 30, no. 7, p. e13316, 2018.

[20] Q. M. Xue, N. Li, and Z. S. Liu, "Efficacy of electroacupuncture in the treatment of functional constipation: a randomized controlled pilot trial," China Journal of Integrated Medicine, vol. 21, no. 1, pp. 459-463, 2015.

[21] C. Liang, K. Wang, and B. Xu, "Electroacupuncture at acupoint ST37 (Shangjuxu) improves function of the enteric nervous system in a novel mouse constipation model," $B M C$ Complementary and Alternative Medicine, vol. 16, no. 1, p. 392, 2016.

[22] X. Zhu, Z. Liu, H. Qu et al., "The effect and mechanism of electroacupuncture at LI11 and ST37 on constipation in a rat model," Acupuncture in Medicine, vol. 34, no. 3, pp. 194-200, 2016.

[23] F. Xiong, Y. Wang, S. Q. Li, M. Tian, C.-H. Zheng, and G.-Y. Huang, "Clinical study of electro-acupuncture treatment with different intensities for functional constipation patients," Journal of Huazhong University of Science and Technology [Medical Sciences], vol. 34, no. 5, pp. 775-781, 2014.

[24] J. D. Z. Chen, M. Ni, and J. Yin, "Electroacupuncture treatments for gut motility disorders," Neurogastroenterol Motil, vol. 30, no. 7, p. e13393, 2018.

[25] C. Liang, K. Y. Wang, and M. R. Gong, "Electro-acupuncture at ST37 and ST25 induce different effects on colonic motility via the enteric nervous system by affecting excitatory and inhibitory neurons," Neurogastroenterology \& Motility, vol. 30, no. 7, p. e13318, 2018.

[26] G. P. T. Higgins, D. G. Altman, and P. C. Gøtzsche, "The cochrane collaboration's tool for assessing risk of bias in randomized trials," BMJ, vol. 343, no. 1, p. d5928, 2011.

[27] D. A. Drossman and D. L. Dumitrascu, "Rome III:new standard for functional gastrointestinal disorders," Journal of Gastrointestinal and Liver Diseases :JGLD, vol. 15, no. 3, pp. 237-241, 2006.

[28] A. R. Jadad, R. A. Moore, D. Carroll et al., "Assessing the quality of reports of randomized clinical trials: is blinding necessary?" Controlled Clinical Trials, vol. 17, no. 1, pp. 1-12, 1996.

[29] The Cochrane Collaboration, Copenhagen: The Nordic Cochrane Centre, The Cochrane Collaboration, London, UK, 2011, https:/training. cochrane.org/online-learning/core-software-cochrane-reviews/revman/.

[30] F. Agachan, T. Chen, J. Pfeifer, P. Reissman, and S. D. Wexner, "A constipation scoring system to simplify evaluation and management of constipated patients," Diseases of the Colon \& Rectum, vol. 39, no. 6, pp. 681-685, 1996.

[31] W. Zhang, "Evaluation of therapeutic effect and safety of deep acupuncture at Tianshu (ST25) for treatment of slow transit constipation," Journal of Traditional Chinese Medicine, vol. 2, no. 1, pp. 105-107, 2006.

[32] L. Chen, A Clinical Observation on the Timeliness of Deep Acupuncture at Tianshu (St25) in the Treatment of Chronic Functional Constipation, Nanjing University of Chinese Medicine, Nanjing, China, 2010.

[33] D. L. Yang and Z. S. Liu, "Observation on the therapeutic effect of deep acupuncture on Tianshu in the treatment of functional constipation," Beijing Journal of Traditional Chinese Medicine, vol. 5, no. 1, pp. 366-368, 2010.

[34] R. Z. Lin, Clinical Study of Deep Acupuncture at Tianshu (St25) for Treatment of Chronic Functional Constipation, Nanjing University of Chinese Medicine, Nanjing, China, 2010.

[35] W. M. Peng, P. P. Qin, and J. Guo, "Efficacy and safety of individualized deep needling at Tianshu in the treatment of colonic slow transit constipation," Jiangsu Journal of Traditional Chinese Medicine, vol. 7, no. 1, pp. 43-45, 2010.

[36] X. Wan, Observation on the Long-Term Effect of Deep Needling at Tianshu Combined with Electro-Acupuncture in the Treatment of Functional Constipation, Nanjing University of Chinese Medicine, Nanjing, China, 2010.

[37] Z. S. Liu, L. L. Wang, N. Li, J. H. Sun, and L. P. Wang, "Individualized deep acupuncture of Tianshu in the treatment of functional constipation: a multicenter randomized controlled trial of efficacy and safety," China Association of Acupuncture Moxibustion, vol. 1, no. 10, pp. 187-191, 2011.

[38] L. Wang, W. N. Peng, and J. Guo, "Improving effects of clinical symptoms and therapeutic satisfaction on slow transit constipation patients through deep electro acupuncture at Tianshu (ST25)," Journal of Clinical Acupuncture Moxibustion, vol. 2, no. 1, pp. 1-5, 2013.

[39] J. Wu, B. Liu, N. Li et al., "Effect and safety of deep needling and shallow needling for functional constipation," Medicine, vol. 93, no. 28, p. e284, 2014.

[40] Y. L. Liao, "Evaluation of the efficacy and safety of deep needling at Tianshu in the treatment of slow transit constipation of colon," Health Must-Read Magazine, vol. 1, no. 1, pp. 190-191, 2018.

[41] W. Voskuijl, F. Lorijn, and W. Verwijs, "PEG 3350 (Transipeg) versus lactulose in the treatment of childhood functional constipation: a double blind, randomised, controlled, multicentre trial," Gut, vol. 53, no. 1, pp. 1590-1594, 2004.

[42] A. Szilagyi, "Lactose-a potential prebiotic," Alimentary Pharmacology and Therapeutics, vol. 16, no. 9, pp. 1591-1602, 2002.

[43] A. Attar, M. Lémann, A. Ferguson et al., "Comparison of a low dose polyethylene glycol electrolyte solution with lactulose for treatment of chronic constipation," Gut, vol. 44, no. 2, pp. 226-230, 1999.

[44] Y. Feng and Y. Xiang, "Study on the regularity of acupoint selection in the treatment of constipation with acupuncture," Journal of Clinical Acupuncture Moxibustion, vol. 19, no. 10, pp. 3-5, 2003.

[45] L. G. Liu and J. Gu, A Survey of Acupuncture Books for Acute Diseases, Shanghai Science and Technology Publishing House, Shanghai, China, 2000.

[46] Q. G. Qin, "Study on the Regulating Effect and Neural Mechanism of Acupuncture on Intestinal Movement in 
Different States," Hubei University of Chinese Medicine, Hubei, China, 2013.

[47] Z. Yang, "Observation of the effect of acupuncture on gastrointestinal motor function after acupuncture by body surface electrogastrogram," Acupuncture Research, vol. 11, no. 4, p. 20, 1986.

[48] Y. D. Hu and L. Lin, "Cajai Interstitial cells and slow Transport constipation," Internal Journal of Internal Medicine, vol. 31, no. 11, pp. 503-506, 2004.

[49] J. Chen and P. Bo, "Research status and trend of Cajal Interstitial cells and slow transport constipation," Internal Journal of Digestive Diseases, vol. 28, no. 5, pp. 383-412, 2008.

[50] G. L. Lyford, C. L. He, and E. Soffer, "Pan-colonic decrease in interstitial cells of Cajal in patients with slow transit constipation," Gut, vol. 51, no. 4, pp. 496-501, 2002.

[51] S. R. Huang, "Post-effect of acupuncture analgesia, acupuncture tolerance and acupuncture frequency," China Journal of Pain and Medicine, vol. 12, no. 6, pp. 360-362, 2006. 